\title{
CRISPR-Cas Controls Cryptic Prophages
}

\author{
Sooyeon Song ${ }^{1,2,3^{*}}$ and Thomas K. Wood $^{1 *}$ \\ ${ }^{1}$ Department of Chemical Engineering, Pennsylvania State University, \\ University Park, Pennsylvania, 16802-4400, USA \\ Departments of Animal Science ${ }^{2}$ and Agricultural Convergence Technology ${ }^{3}$, Jeonbuk National \\ University, 587 Baekje-Daero, Deojin-Gu, Jeonju-Si, Jellabuk-Do, 54896, South Korea
}

*For correspondence. E-mail songsy@jbnu.ac.kr, tuw14@psu.edu

Tel. (+)1 814-863-4811; Fax (1) 814-865-7846

\section{Running title: CRISPR-Cas tames cryptic prophages.}

Keywords: CRISPR-Cas, persisters, cryptic prophage 
CRISPR-Cas via deletion of cas2, which encodes one of the two conserved CRISPR-Cas proteins, reduces growth by $40 \%$, increases cell death by $700 \%$, and prevents persister cell resuscitation; hence, CRISPRCas serves to inhibit the remaining deleterious effects of these cryptic prophages. Consistently, seven of the 13 E. coli spacers contain matches to the cryptic prophages, and, after excision, CRISPR-Cas cleaves cryptic prophage CP4-57 and DLP-12 DNA. Moreover, we determine that the key genes in these cryptic prophages that CRISPR-Cas represses by cleaving the excised DNA include lysis protein YdfD of Qin and lysis protein RzoD of DLP-12. Therefore, we report the novel results that (i) CRISPR-Cas is active in E. coli and (ii) CRISPR-Cas is used to tame cryptic prophages; i.e., unlike with active lysogens, CRISPR-Cas and cryptic prophages may stably exist. 


\section{INTRODUCTION}

Along with restriction/modification ${ }^{1}$ and toxin/antitoxin (TA) systems ${ }^{2}$, prokaryotes utilize clustered, regularly-interspaced, short palindromic repeats (CRISPR) and CRISPR-associated (Cas) ${ }^{3}$ proteins to combat phages. These systems are interrelated in that we found some Cas proteins are derived from TA systems; for example, Sulfolobus solfataricus Cas2 is structurally similar to antitoxin GhoS of the Escherichia coli GhoT/GhoS TA system ${ }^{4}$. In addition, in a manner similar to our discovery ${ }^{2}$ that toxins of TA systems inhibit phage by degrading host mRNA after the toxin is activated by the attacking phage when the phage shuts down transcription (e.g., Hok/Sok inhibits T4 phage), some Cas proteins induce host dormancy rather than degrading phage DNA to inhibit phage propagation ${ }^{5}$. Also, TA systems have been found to stabilize CRISPR-Cas systems by making them addictive to the host ${ }^{6}$.

Although CRISPR-Cas systems exclude both external lytic and temperate (lysogenic) phages ${ }^{7}$, either the cell dies or the phage is lost ${ }^{7,8}$. Also, the class I-E ${ }^{3}$ CRISPR-Cas system of E. coli is not related to immunity for external phages ${ }^{9}$ and is thought to be inactive in the wild-type $\operatorname{strain}^{10}$, due to repression by H-NS, although it is functional when induced ${ }^{11}$. To date, the relationship of CRISPR-Cas to cryptic prophages; i.e., those phage remnants that are unable to form lytic particles, has not been investigated.

Up to $20 \%$ of bacterial genomes may contain stable phage DNA ${ }^{12}$, and for $E$. coli, we discovered that its nine cryptic prophages are not genomic junk but instead encode genes for proteins that increase resistance to sub-lethal concentrations of quinolone and $\beta$-lactam antibiotics as well as protect the cell from osmotic, oxidative, and acid stresses ${ }^{13}$. Although these cryptic prophages do not help a subpopulation of cells weather extreme stress by entering into the dormant state known as persistence ${ }^{14}$, these phage remnants facilitate the resuscitation of these persister cell via nutrient sensing ${ }^{15}$. Therefore, the bacterial cell can capture the genome of its former parasite to both combat stress ${ }^{13}$ as well as to revive from dormancy ${ }^{15}$.

Here we explore the role of the native E. coli CRISPR-Cas systems in the regulation of cryptic prophages. We find that the CRISPR-Cas system is required for inhibiting these phage fossils since, if CRISPR-Cas is inactivated, cells die due to activation of the lysis proteins YdfD of Qin and RzoD from 
DLP-12. Hence, we discovered CRISPR-Cas is active in E. coli and serves to regulate its phage fossils.

\section{RESULTS}

CRISPR-Cas increases growth. To inactivate CRISPR-Cas in E. coli, the cas2 deletion was chosen since Cas2 is conserved in almost all CRSIPR-Cas systems ${ }^{16}$ as part of the adaptor complex and serves as the structural scaffold of the Cas1/Cas2 complex which cleaves the source, protospacer DNA, and the CRISPR array ${ }^{17}$. We found the E. coli $\mathrm{K}-12$ cas2 mutant grows $40 \%$ slower in rich medium compared to the wildtype strain (specific growth rate of $0.79 \pm 0.21 / \mathrm{h}$ vs. $1.3 \pm 0.11 / \mathrm{h}$, respectively). Similarly, in minimal glucose $(0.4 \mathrm{wt} \%)$ medium, deletion of cas 2 also reduces growth by $33 \%(0.42 \pm 0.02 / \mathrm{h}$ vs. $0.62 \pm 0.02 / \mathrm{h}$, respectively).

CRISPR-Cas increases single-cell resuscitation. Moreover, since CRSIPR-Cas increases growth, we tested for its effect on persister cell resuscitation using single-cell microscopy. Persister cell resuscitation is germane in that the dormant cells are highly stressed and have limited resources for their revival via activation of hibernating ribosomes ${ }^{18,19}$; for example, we have shown inhibiting ATP synthesis leads to a 5,000 -fold increase in persister cell formation ${ }^{20}$. Since we discovered facile means for converting the whole population of cells into persister cells ${ }^{18-20}$ that has been used by at least 17 independent labs to date with various bacterial species ${ }^{21}$, these stressed cells are an excellent model for testing the effects of CRISPRCas on E. coli physiology.

Here, we found inactivation of CRISPR-Cas via cas2 nearly completely prevents persister cell resuscitation, while the wild-type cell has 52\% resuscitation (Fig. 1A, Table S1). Hence, CRSIPR-Cas is active in E. coli and plays key roles in its growth and recovery from extreme stress.

60 CRISPR-Cas prevents cell death. To explore how CRISPR-Cas increases growth (including persister cell resuscitation), we checked for death in resuscitating persister cells of the cas2 deletion strain using the Live/Dead stain. We found that inactivating CRISPR-Cas leads to a 7-fold increase in death in resuscitating cells (Fig. 1B, Table S2). In addition, there were 34-fold more cells termed "ghosts"22 that lack cytosolic material (Fig. S1) and are likely dead but have intact membranes so are not stained by the propidium iodide 
dye we use. Corroborating these results, there was 11-fold and 5-fold more death for stationary- and exponential-phase cells, respectively, when CRISPR-Cas was inactivated. These results indicate inactivating CRISPR-Cas leads to cell death.

CRISPR-Cas increases growth by repressing cryptic prophage genes. We hypothesized that since CRISPR-Cas systems inhibit some phage lysogens ${ }^{7,8}$, the $E$. coli system may be preventing cell death by repressing some of the cryptic prophage genes. To test this hypothesis, we first examined the E. coli CRISPR-Cas system for spacers related to the nine cryptic prophages. E. coli K-12 CRISPR-Cas contains 13 spacers $^{11,23}$, each containing 32 or $33 \mathrm{nt}^{24}$, between iap and cas2. Between the 1429 -nt repeat sequences (5'-GTGTTCCCCGCATCAGCGGGGATAAACCG), we found that six of the 13 spacers contain 12 to 16 nt of perfect matches to seven of the nine cryptic prophages (DLP-12, CP4-57, CPS-53, CP4-6, Rac, Qin,

75 and e14) (Fig. 2A). In general, spacer lengths vary from 21 to $72 \mathrm{nt}^{25}$ with perfect complementarity of 6 to $12 \mathrm{nt}^{10}$. Furthermore, the two predicted protospacer adjacent motif (PAM) sites for cryptic phage DLP-12, 5'-GAA (consensus I-E site) and 5'CAT (Fig. S2), support rapid and reduced interference in E. coli, respectively ${ }^{26}$. Moreover, we found these spacers cannot be deleted, which shows they are essential for $E$. coli K-12. Together, these results suggest CRISPR-Cas regulates the E. coli cryptic prophages. Cas suggested that E. coli CRISPR-Cas suggested may be repressing some of the cryptic prophage lysis genes; hence, we checked for derepression of the cryptic prophage lysis genes in these seven cryptic prophages, specifically ydfD (Qin), hokD (Qin), ypjF (CP4-57), essD (DLP-12), and rzoD (DLP-12) via quantitative reverse transcription polymerase chain reaction (qRT-PCR). We found that inactivation of CRISPR-Cas via the cas2 deletion results in activation of $y d f D$ by 35 -fold and activation of rzoD by 183 fold in resuscitating persister cells (Fig. 1C). Moreover, rzoD was activated 1024-fold in exponentiallygrowing cells (Fig. 1C). RzoD is a putative DLP-12 lysis lipoprotein that we previously showed was toxic through its interaction with toxin $\mathrm{Hha}^{27}$ of the Hha/TomB TA system ${ }^{28}$. YdfD of Qin cryptic prophage has been shown to lyse cells when induced ${ }^{29}$. Hence, CRSIPR-Cas represses at least two E. coli proteins RzoD and YdfD that can reduce cell growth. 
Excision of DLP-12 is not regulated by CRISPR-Cas. Since inactivation of CRSIPR-Cas leads to extraordinary derepression of the DLP-12 rzoD lysis gene, we checked for increased excision with the cas2 deletion strain using qPCR. We found the cas2 deletion has little impact on DLP-12 excision. We also tested the effect of CRISPR-Cas on two other cryptic prophages with significant excision (CP4-57 and e14) ${ }^{13}$, and also found no effect of deleting cas2 (Table S3). Hence, CRISPR-Cas does not affect cryptic prophage excision.

CRISPR-Cas cleaves DLP-12 and CP4-57 DNA after excision. Since DLP-12 has significant excision in E. coli $^{13}$ but inactivation of CRISPR-Cas does not alter its excision, we hypothesized that CRISPR-Cas repressed lysis gene rzoD by cleaving DLP-12 DNA after excision. Using qRT-PCR with resuscitating

100 persister cells, we found that the cas2 deletion leads to 40-fold increase in DLP-12 DNA that includes spacer 3 as well a 184-fold increase in CP4-57 DNA that includes spacer 6 (Fig. 2B). Therefore, CRISPRCas actively cleaves excised cryptic prophage DNA to regulate the lysis genes of the captured phage foe.

\section{DISCUSSION}

Our results reveal a new role for CRISPR-Cas systems: regulation of phage fossils. The evidence for this includes that inactivating CRISPR-Cas by deleting cas2 (i) reduces growth by 40\%, (ii) nearly eliminates resuscitation from the persister state (Fig. 2), (iii) causes ghost cell formation and cell death (Fig. 1), (iv) derepresses the cryptic prophage lysis genes $y d f D$ and $r z o D$, and (v) increases CP4-57 and DLP-12 cryptic prophage DNA after their excision. Since there is no change in excision of DLP-12 upon inactivating CRISPR-Cas, our results show the mechanism for regulating the lysis genes $y d f D$ and $r z o D$ of the cryptic 110 prophages is via CRISPR-Cas cleavage of the excised prophages. Supporting this, the E. coli K-12 class 1E CRISPR-Cas system cleaves dsDNA ${ }^{30}$. What remains to be determined is the mechanism by which CRISPR-Cas cleaves only the excised cryptic prophage rather than genomic DNA; perhaps this is related to the relatively inefficient PAM and seeding regions for some of the spacers (Fig. S2).

This new function for CRISPR-Cas is likely general and may explain why many species appear to have 115 inactive CRISPR-Cas systems as was previously thought for E. coli ${ }^{10}$; i.e., instead of protecting cells from 
external phages, CRISPR-Cas systems may also control resident cryptic prophages which are prevalent. Critically, our results provide the first example where it is beneficial for the host to have an active CRISPRCas system that targets inactive integrated phages (i.e., cryptic prophages) since previous reports show targeting active temperate phages is deleterious; i.e., either the cell dies or the phage is $\operatorname{lost}^{7,8,31}$. Since $E$.

120 coli cryptic prophages like rac have been present in its genome for 4.5 million years ${ }^{32}$, the active K-12 CRISPR-Cas system is stable with the cryptic prophages; in fact, there has been little change in the E. coli spacers for at least 42,000 years $^{33}$.

Our results also indicate that, although the cryptic prophages are stable and the cell makes use of the genetic tools encoded by its former foe to combat myriad stresses ${ }^{13}$ and to sense nutrients prior to exiting

125 the persister state ${ }^{15}$, the source of these tools must be elegantly regulated by CRISPR-CAS since they often harbor deleterious membrane lysis proteins like YdfD and RzoD. Similarly, host Rho has been found recently to silence cryptic prophage toxin/antitoxin systems through transcription termination ${ }^{34}$, and H-NS silences cryptic prophages through 65 binding sites ${ }^{35}$. Therefore, phages may be captured by the host, but they must be tamed, and this now includes repression by CRISPR-Cas.

\section{MATERIALS AND METHODS}

Bacteria and growth conditions. Bacteria (Table S4) were cultured in lysogeny broth ${ }^{36}$ at $37^{\circ} \mathrm{C}$. pCA24Nbased plasmids ${ }^{37}$ were retained in overnight cultures via chloramphenicol (30 $\left.\mu \mathrm{g} / \mathrm{mL}\right)$, and kanamycin (50 $\mu \mathrm{g} / \mathrm{mL}$ ) was used for deletion mutants, where applicable.

Spacer knockout. To attempt to delete the CRISPR-Cas spacer region, the one-step inactivation method 135 for single gene deletions ${ }^{38}$ was utilized in which primers that included the sequences flanking the spacer region (Table S5) were used with plasmid pKD4 to allow for insertion of the kanamycin resistance cassette $(1.5 \mathrm{~kb})$ and FRT sites.

Persister cells. Exponentially-growing cells (turbidity of 0.8 at $600 \mathrm{~nm}$ ) were converted nearly completely to persister cells ${ }^{18,20}$ by adding rifampicin $(100 \mu \mathrm{g} / \mathrm{mL})$ for $30 \mathrm{~min}$ to stop transcription, centrifuging, and adding LB with ampicillin (100 $\mathrm{gg} / \mathrm{mL})$ for $3 \mathrm{~h}$ to lyse non-persister cells. To remove ampicillin, cells were 
washed twice with $0.85 \% \mathrm{NaCl}$ then re-suspended in $0.85 \% \mathrm{NaCl}$. Persister concentrations were enumerated via a drop assay ${ }^{39}$.

Single-cell persister resuscitation. Persister cells $(5 \mu \mathrm{L})$ were added to $1.5 \%$ agarose gel pads containing M9 glucose (0.4 wt\%) medium ${ }^{40}$, and single-cell resuscitation was visualized at $37^{\circ} \mathrm{C}$ via a light microscope

145 (Zeiss Axio Scope.A1, bl_ph channel at $1000 \mathrm{~ms}$ exposure). For each condition, at least two independent cultures were used with 150 to 300 individual cells used per culture.

Membrane integrity assay. To determine membrane integrity, the persister cells were analyzed with the LIVE/DEAD BacLight Bacterial Viability Kit (Molecular Probes, Inc., Eugene, OR, catalog number L7012). The fluorescence signal was analyzed via a Zeiss Axioscope.A1 using excitation at $485 \mathrm{~nm}$ and emission at $530 \mathrm{~nm}$ for green fluorescence and using excitation at $485 \mathrm{~nm}$ and emission at $630 \mathrm{~nm}$ for red fluorescence.

qRT-PCR. To quantify transcription from the cryptic prophage lytic genes, RNA was isolated from persister cells that were resuscitated by adding M9 glucose (0.4\%) medium for 10 min then washed with $0.85 \% \mathrm{NaCl}$ and from exponential cells grown to a turbidity of 0.8 ; samples were cooled rapidly using ethanol/dry ice in the presence of RNA Later. RNA was isolated using the High Pure RNA Isolation Kit (Roche). The following qRT-PCR thermocycling protocol was used with the iTaq ${ }^{\mathrm{TM}}$ universal $\mathrm{SYBR}^{\circledR}$ Green One-Step kit (Bio-Rad): $95^{\circ} \mathrm{C}$ for $5 \mathrm{~min} ; 40$ cycles of $95^{\circ} \mathrm{C}$ for $15 \mathrm{~s}, 60^{\circ} \mathrm{C}$ for $1 \mathrm{~min}$ for two replicate reactions for each sample/primer pair. The annealing temperature was $60^{\circ} \mathrm{C}$ for all primers (Table S5). qPCR. To quantify prophage excision and the levels of DNA flanking the CRISPR-Cas cleavage sites, total DNA (100 ng) was isolated from exponentially-growing and persister resuscitating cells using an UltraClean Microbial DNA Isolation Kit (Mo Bio Laboratories). Excised cryptic prophage was quantified using primers for each prophage excisionase (Table S5) that only yield a PCR product upon prophage excision, and the relative amount of each target gene was determined using reference gene purM. The level of cryptic prophage flanking the CRISPR-Cas cleave site was quantified using primers that flank each site

165 (Table S5). The qPCR reaction performed using CFX96 Real Time System. The reaction and analysis was conducted using the StepOne Real-Time PCR System (Bio-Rad). 


\section{ACKNOWLEDGEMENTS}

This work was supported by funds derived from the Biotechnology Endowed Professorship at the

Pennsylvania State University for TKW and from the National Research Foundation of Korea (NRF) grant

170 from the Korean Government (NRF-2020R1F1A1072397) for SYS. We appreciate the feedback of Dr.

Joseph Wade on the PAM sequences. The authors have no conflicts of interest.

\section{REFERENCES}

1. Vasu, K. \& Nagaraja, V. Diverse Functions of Restriction-Modification Systems in Addition to Cellular Defense. Micro Molecr Biol Rev 77, 53-72 (2013).

2. Pecota, D. C. \& Wood, T. K. Exclusion of T4 Phage by the hok/sok Killer Locus from Plasmid R1. $J$ Bacteriol 178, 2044-2050 (1996).

3. Makarova, K. S. et al. Evolutionary classification of CRISPR-Cas systems: a burst of class 2 and derived variants. Nat Rev Microbiol 18, 67-83 (2020).

4. Wang, X. et al. A Novel Type V TA System Where mRNA for Toxin GhoT is Cleaved by Antitoxin GhoS. Nat Chem Biol 8, 855-861 (2012).

5. Meeske, A. J., Nakandakari-Higa, S. \& Marraffini, L. A. Cas13-induced cellular dormancy prevents the rise of CRISPR-resistant bacteriophage. Nature 570, 241-245 (2019).

6. Li, M. et al. Toxin-antitoxin RNA pairs safeguard CRISPR-Cas systems. Science 372, eabe5601 (2021).

7. Goldberg, G. W. et al. Incomplete prophage tolerance by type III-A CRISPR-Cas systems reduces the fitness of lysogenic hosts. Nat Commun 9, 61 (2018).

8. Edgar, R. \& Qimron, U. The Escherichia coli CRISPR System Protects from $\lambda$ Lysogenization, Lysogens, and Prophage Induction. J Bacteriol 192, 6291-6294 (2010).

9. Touchon, M. et al. CRISPR Distribution within the Escherichia coli Species Is Not Suggestive of Immunity-Associated Diversifying Selection. J Bacteriol 193, 2460-2467 (2011).

10. Shmakov, S. A. et al. The CRISPR Spacer Space Is Dominated by Sequences from Species-Specific Mobilomes. mBio 8, e01397-01317 (2017).

11. Pougach, K. et al. Transcription, processing and function of CRISPR cassettes in Escherichia coli. Mol Microbiol 77, 1367-1379 (2010).

12. Casjens, S. Prophages and bacterial genomics: what have we learned so far? Mol Microbiol 49, 277300 (2003).

13. Wang, X. et al. Cryptic prophages help bacteria cope with adverse environments. Nat Commun 1, 147 (2010).

14. Wood, T. K. \& Song, S. Forming and waking dormant cells: The ppGpp ribosome dimerization persister model. Biofilm 2, 100018 (2020).

15. Song, S. et al. Escherichia coli Cryptic Prophages Sense Nutrients to Control Persister Cell Resuscitation. bioRxiv on-line, 2021.2004.2010.439273 (2021).

16. Watson, B. N. J., Steens, J. A., Staals, R. H. J., Westra, E. R. \& van Houte, S. Coevolution between bacterial CRISPR-Cas systems and their bacteriophages. Cell Host Microbe 29, 715-725 (2021).

17. Koonin, E. V. \& Makarova, K. S. Origins and evolution of CRISPR-Cas systems. Phil Trans. R Soc B 374, 20180087 (2019).

18. Kim, J.-S., Yamasaki, R., Song, S., Zhang, W. \& Wood, T. K. Single Cell Observations Show Persister Cells Wake Based on Ribosome Content. Environ Microbiol 20, 2085-2098 (2018).

19. Yamasaki, R., Song, S., Benedik, M. J. \& Wood, T. K. Persister Cells Resuscitate Using Membrane Sensors that Activate Chemotaxis, Lower cAMP Levels, and Revive Ribosomes. iScience 23, 100792 (2020). 
20. Kwan, B. W., Valenta, J. A., Benedik, M. J. \& Wood, T. K. Arrested protein synthesis increases persister-like cell formation. Antimicrob Agents Chemother 57, 1468-1473 (2013).

21. Song, S. \& Wood, T. K. Are We Really Studying Persister Cells? Environ Microbiol Rep 13, 3-7 (2021).

22. Cheng, H.-Y. et al. Toxin GhoT of the GhoT/GhoS toxin/antitoxin system damages the cell membrane to reduce adenosine triphosphate and to reduce growth under stress. Environ Microbiol 16, 1741-1754 (2014).

23. Militello, K. T. \& Lazatin, J. C. Discovery of Escherichia coli CRISPR sequences in an undergraduate laboratory. Biochem Molec Biol Edu 45, 262-269 (2017).

24. Brouns, S. J. J. et al. Small CRISPR RNAs Guide Antiviral Defense in Prokaryotes. Science 321, 960964 (2008).

25. Barrangou, R. \& Marraffini, Luciano A. CRISPR-Cas Systems: Prokaryotes Upgrade to Adaptive Immunity. Mol Cell 54, 234-244 (2014).

26. Musharova, O. et al. Systematic analysis of Type I-E Escherichia coli CRISPR-Cas PAM sequences ability to promote interference and primed adaptation. Mol Microbiol 111, 1558-1570 (2019).

27. García Contreras, R., Zhang, X.-S., Kim, Y. \& Wood, T. K. Protein Translation and Cell Death: The Role of Rare tRNAs in Biofilm Formation and in Activating Dormant Phage Killer Genes. PLoS ONE 3, e2394 (2008).

28. Marimon, O. et al. An oxygen-sensitive toxin-antitoxin system. Nat Comm 7, 13634 (2016).

29. Masuda, H., Awano, N. \& Inouye, M. ydfD encodes a novel lytic protein in Escherichia coli. FEMS Microbiol Lett 363 (2016).

30. Hochstrasser, M. L. et al. CasA mediates Cas3-catalyzed target degradation during CRISPR RNAguided interference. Proc Natl Acad Sci USA 111, 6618-6623 (2014).

31. Rollie, C. et al. Targeting of temperate phages drives loss of type I CRISPR-Cas systems. Nature (2020).

32. Perna, N. T. et al. Genome sequence of enterohaemorrhagic Escherichia coli O157: H7. Nature 409, 529-533 (2001).

33. Savitskaya, E. et al. Dynamics of Escherichia coli type I-E CRISPR spacers over 42000 years. Molecular Ecol 26, 2019-2026 (2017).

34. Hafeezunnisa, M., Chhakchhuak, P. I. R., Krishnakumar, J. \& Sen, R. Rho-dependent transcription termination regulates the toxin-antitoxin modules of cryptic prophages to silence their expression in Escherichia coli. FEBS Lett on-line (2021).

35. Ishihama, A. \& Shimada, T. Hierarchy of transcription factor network in Escherichia coli K-12: H-NSmediated silencing and Anti-silencing by global regulators. FEMS Microbiol Rev (2021).

36. Bertani, G. Studies on Lysogenesis .1. The Mode of Phage Liberation by Lysogenic Escherichia coli. J Bacteriol 62, 293-300 (1951).

37. Kitagawa, M. et al. Complete set of ORF clones of Escherichia coli ASKA library (A Complete Set of E. coli K-12 ORF Archive): Unique Resources for Biological Research. DNA Res 12, 291-299 (2005).

38. Datsenko, K. A. \& Wanner, B. L. One-step inactivation of chromosomal genes in Escherichia coli K12 using PCR products. Proc Natl Acad Sci USA 97, 6640-6645 (2000).

39. Donegan, K., Matyac, C., Seidler, R. \& Porteous, A. Evaluation of methods for sampling, recovery, and enumeration of bacteria applied to the phylloplane. Appl Environ Microbiol 57, 51-56 (1991).

40. Rodriguez, R. L. \& Tait, R. C. Recombinant DNA Techniques: An Introduction. (Benjamin/Cummings Publishing, 1983).

41. Baba, T. et al. Construction of Escherichia coli K-12 in-frame, single-gene knockout mutants: the Keio collection. Mol Syst Biol 2, 20060008 (2006).

42. Kitagawa, M. et al. Complete set of ORF clones of Escherichia coli ASKA library (a complete set of E. coli K-12 ORF archive): unique resources for biological research. DNA Res 12, 291-299 (2005). 


\section{FIGURE LEGENDS}

Fig. 1. Inactivating CRISPR-Cas eliminates persister cell resuscitation by activating cryptic prophage lytic proteins, causing cell death. (A) Single cell persister resuscitation for wild-type BW25113 and the cas2 mutant after 6 hours on $0.4 \mathrm{wt} \%$ glucose. Black arrows indicate cells that resuscitate, and the scale bar indicates $10 \mu \mathrm{m}$. Cells were observed using light microscopy (Zeiss Axio Scope.A1). Representative results from two independent cultures are shown, and tabulated cell numbers are shown in Table S1. (B) LIVE/DEAD staining of resuscitating persister cells shows the cas2 mutation causes cell death. DF is dark field, SYTO9 is a membrane permeable stain for nucleic acids (green), and PI is propidium iodide, which is a membrane impermeable stain for the nucleic acids of dead cells (red). Tabulated cell numbers are shown in Table S2. (C) The cas2 mutation derepresses cryptic prophage lysis genes $y d f D$ (in resuscitating persister cells) and $r z o D$ (in both resuscitating persister cells and exponentially-growing cells). Lytic genes from five cryptic prophages were checked by qRT-PCR: ydfD (Qin), hokD (Qin), ypjF (CP4-57), essD (DLP-12), and $r z o D(\mathrm{DLP}-12)$.

Fig. 2. Inactivating CRISPR-Cas increases excised cryptic prophage DNA. (A) The 14 repeat (R, hexagon) and 13 spacer (squares) sequences of the CRISPR-Cas system (from the iap to cas2 part of the E. coli genome) showing the cryptic prophage spacer matches (red text) and prophage DNA protospacer sequences (blue text) which includes matches to seven of the nine cryptic prophages (DLP-12, CP4-57, CPS-53, CP4-6, rac, Qin, and e14). The potential PAM sequences are shown in Fig. S2. (B) The cas2 mutation increases cryptic prophage excised DNA that flanks the cleavage sites, as determined by qPCR. 
(A)

BW25113

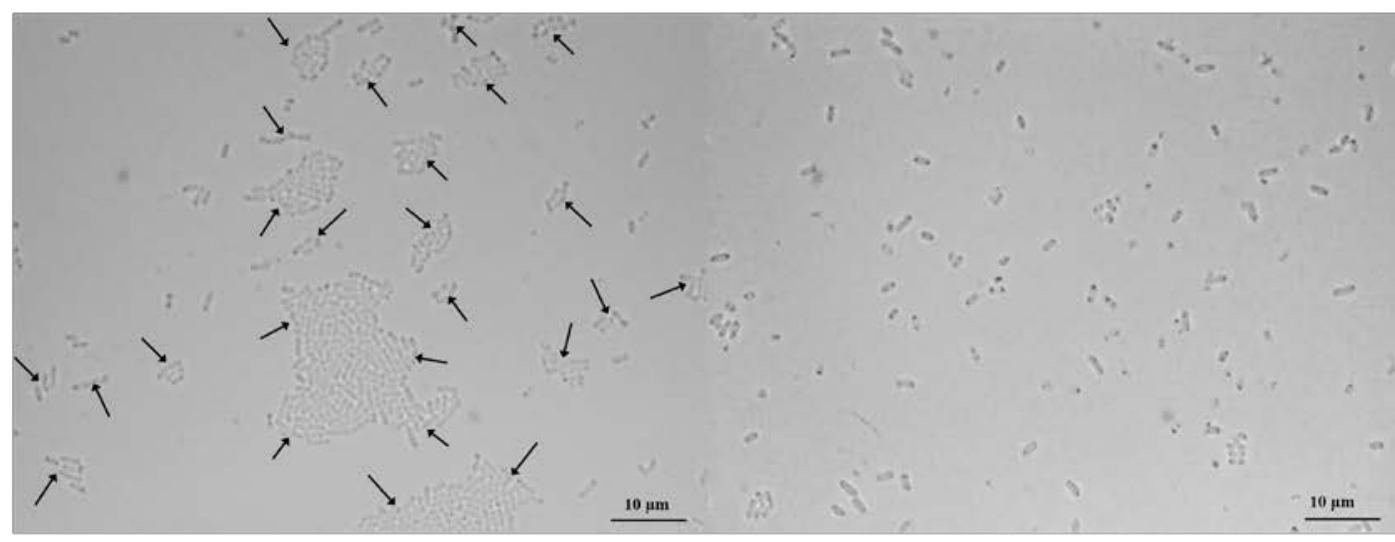

(B)

B)

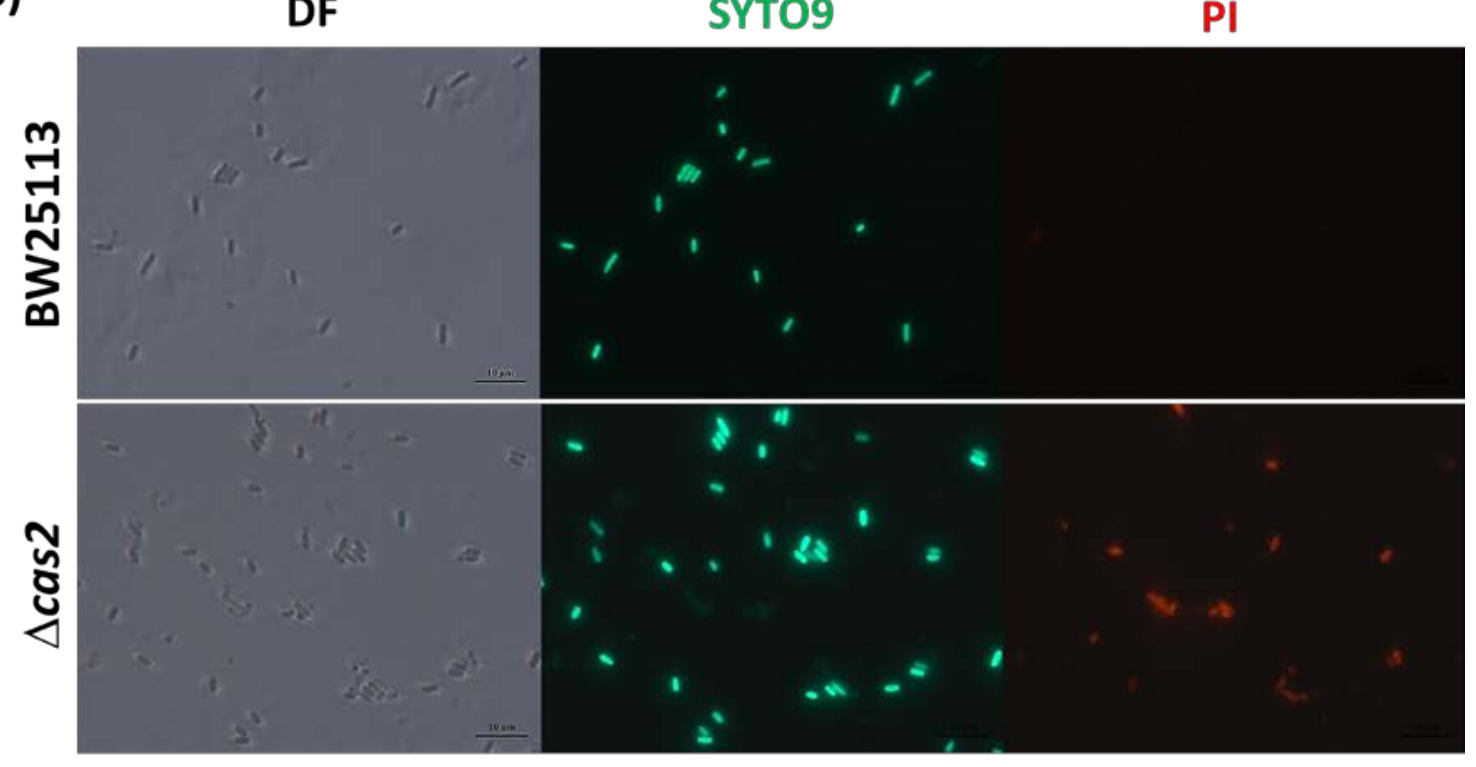

(c)

\section{Exponentially-growing cells}

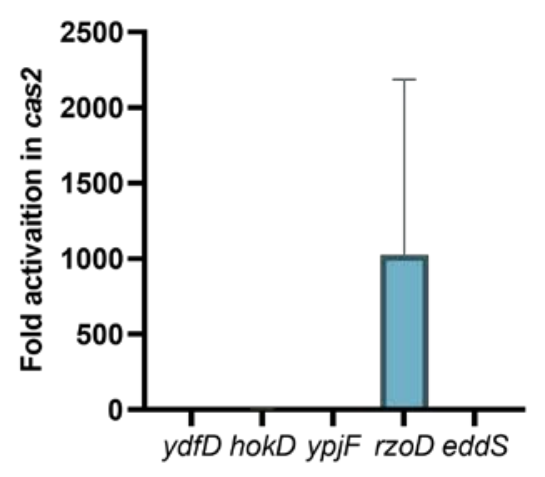

\section{$\Delta$ cas2}

PI

\section{PI}

Resuscitating persister cells

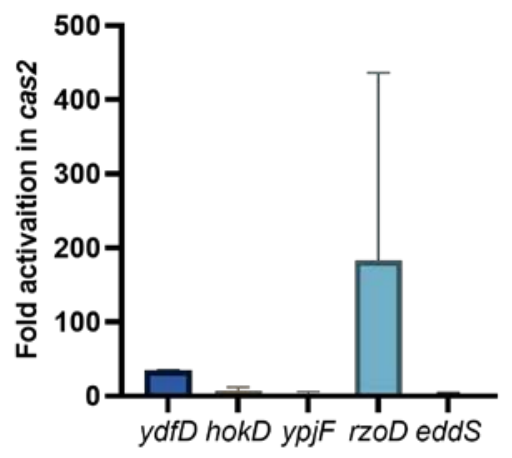

Figure 1 
(A)

Spacer Sequence

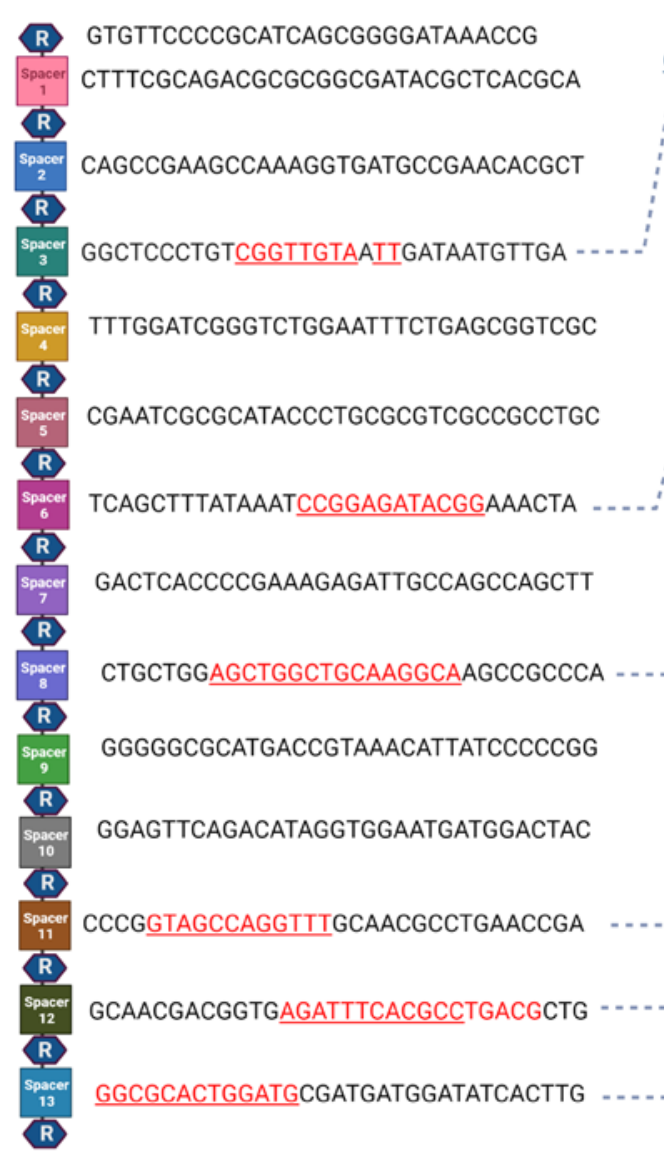

Cryptic prophage Gene

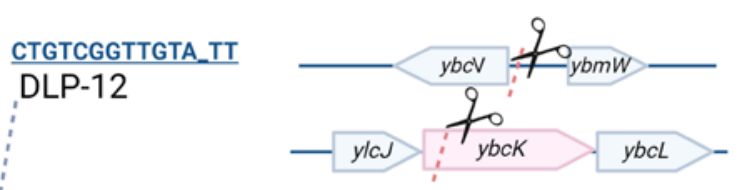

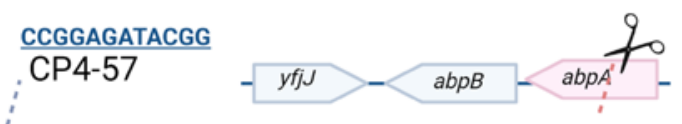
CP4-57

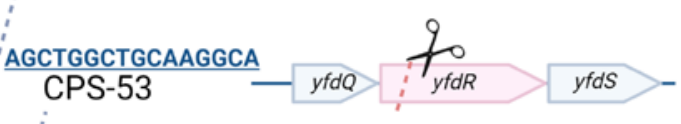

;

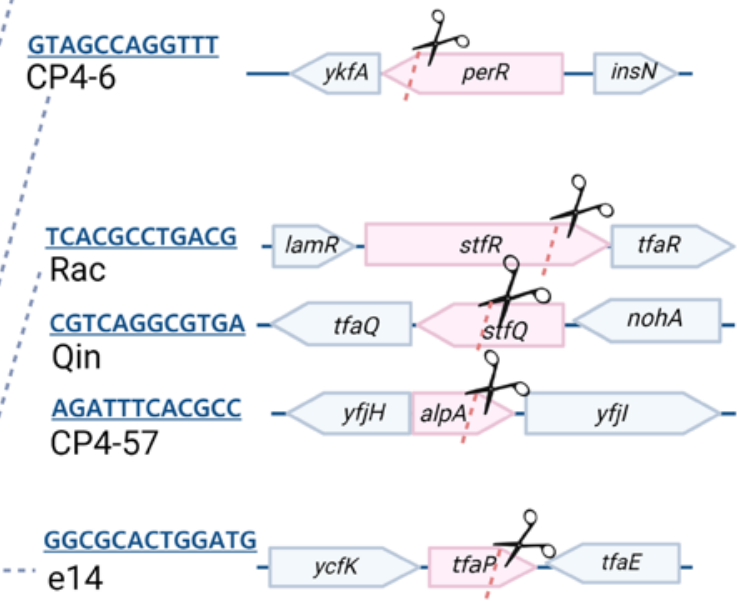

(B)

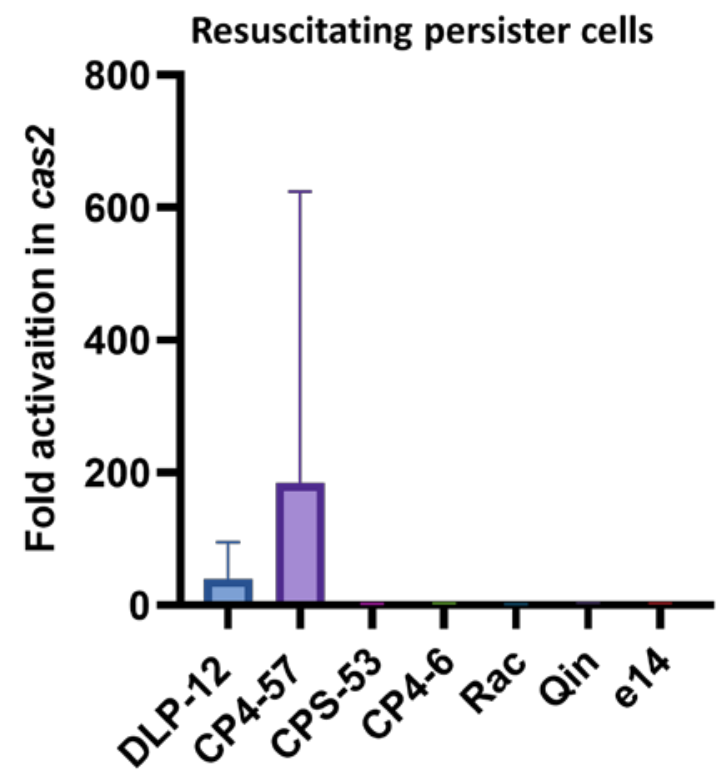

Figure 2 


\title{
Supporting Information
}

\section{CRISPR-Cas Controls Cryptic Prophages}

\author{
Sooyeon Song ${ }^{1,2^{*}}$ and Thomas K. Wood ${ }^{1 *}$ \\ ${ }^{1}$ Department of Chemical Engineering, Pennsylvania State University, \\ University Park, Pennsylvania, 16802-4400, USA \\ Departments of Animal Science ${ }^{2}$ and Agricultural Convergence Technology ${ }^{3}$, Jeonbuk National \\ University, 587 Baekje-Daero, Deojin-Gu, Jeonju-Si, Jellabuk-Do, 54896, South Korea
}

*For correspondence. E-mail songsy@jbnu.ac.kr, tuw14@psu.edu

Tel. (+)1 814-863-4811; Fax (1) 814-865-7846 
Table S1. Inactivating CRISPR-Cas eliminates persister cell resuscitation on glucose agarose gel pads. Single persister cells were observed using light microscopy (Zeiss Axio Scope.A1). The total number and waking number of persister cells are shown after 6 hours on 0.4 wt\% glucose. Fold-change in waking is relative to BW25113. These results are the combined observations from two independent experiments (independent culture results separated by “/”), and standard deviations are shown. The microscope images are shown in Fig. 1.

\begin{tabular}{ccccc}
\hline & $\begin{array}{c}\text { Total } \\
\text { cells }\end{array}$ & $\begin{array}{c}\text { Waking } \\
\text { cells }\end{array}$ & \% waking & $\begin{array}{c}\text { Fold- } \\
\text { change }\end{array}$ \\
\hline BW25113 & $504 / 382$ & $199 / 248$ & $52 \pm 18$ & 1 \\
$\Delta$ cas2 & $516 / 677$ & $6 / 15$ & $1.7 \pm 0.7$ & -30.9 \\
\hline
\end{tabular}


Table S2. Inactivating CRISPR-Cas causes cell death and ghost cell formation. Persister cells were washed with PBS twice, resuscitated by M9 $0.4 \%$ glucose for $10 \mathrm{~min}$ and stained with LIVE/DEAD reagents. Exponential cells were grown to a turbidity of 0.8 (at $600 \mathrm{~nm}$ ) and stationary cells were grown to turbidity of 2.0. The ghost cells in the persister population were visualized using a Zeiss Axioscope.A1microscope. The results are the combined observations from two independent experiments (independent culture results separated by “””). The microscope images are shown in Fig. 1B, Fig. S1, and Fig. S3.

\begin{tabular}{|c|c|c|c|c|c|c|c|c|}
\hline & Strains & Total cells & $\begin{array}{l}\text { Dead } \\
\text { cells }\end{array}$ & $\begin{array}{l}\text { Ghost } \\
\text { cells }\end{array}$ & $\begin{array}{c}\text { \% Ghost } \\
\text { cells }\end{array}$ & $\begin{array}{c}\text { fold- } \\
\text { change } \\
\text { ghost cells }\end{array}$ & $\begin{array}{c}\% \\
\text { dead }\end{array}$ & $\begin{array}{c}\text { fold- } \\
\text { change } \\
\text { dead cells }\end{array}$ \\
\hline \multirow[t]{2}{*}{ Resuscitated } & BW25113 & $52 / 115$ & $2 / 7$ & $0 / 1$ & 0.6 & 1 & 5.4 & 1 \\
\hline & $\Delta c a s 2$ & $142 / 106$ & $54 / 38$ & $38 / 13$ & 20.6 & 34 & 37.1 & 6.9 \\
\hline \multirow[t]{2}{*}{ Exponential } & BW25113 & $153 / 147$ & $0 / 2$ & - & - & - & 0.7 & 1 \\
\hline & $\Delta$ cas2 & $59 / 40$ & $1 / 2$ & - & - & - & 3.4 & 4.9 \\
\hline \multirow[t]{2}{*}{ Stationary } & BW25113 & $492 / 123$ & $1 / 0$ & & & & 0.1 & 1 \\
\hline & $\Delta c a s 2$ & 1136/1736 & $14 / 18$ & - & - & - & 1.13 & 11.2 \\
\hline
\end{tabular}


Table S3. CRISPR-Cas does not affect cryptic prophage excision in stationary cells.

\begin{tabular}{|c|c|c|c|c|c|c|c|c|}
\hline \multirow{2}{*}{$\begin{array}{l}\text { Gene } \\
\text { Strain }\end{array}$} & \multicolumn{2}{|c|}{$\begin{array}{c}\text { purM } \\
\text { (house keeping } \\
\text { gene) }\end{array}$} & \multicolumn{2}{|c|}{ CP4-57 } & \multicolumn{2}{|c|}{ e14 } & \multicolumn{2}{|c|}{ DLP-12 } \\
\hline & WT & Cas2 & WT & cas2 & WT & cas2 & WT & cas2 \\
\hline \multirow{2}{*}{$\mathrm{CT}$} & 9.30 & 9.70 & 30.45 & 30.37 & 16.30 & 17.45 & 30.58 & 30.05 \\
\hline & \pm 0.13 & \pm 0.18 & \pm 1.73 & \pm 0.68 & \pm 0.06 & \pm 0.51 & \pm 0.49 & \pm 0.5 \\
\hline \multirow{2}{*}{$\Delta \mathrm{CT}$} & & & 21.16 & 20.67 & 7.00 & 8.16 & 21.29 & 20.35 \\
\hline & & & \pm 1.73 & \pm 0.70 & \pm 0.15 & \pm 0.54 & \pm 0.51 & \pm 0.53 \\
\hline \multirow{2}{*}{$\Delta \Delta \mathrm{CT}$} & & & & -0.48 & & 1.16 & & -0.94 \\
\hline & & & & \pm 0.7 & & \pm 0.54 & & \pm 0.53 \\
\hline fold & & & & 1.40 & & -2.23 & & 1.92 \\
\hline
\end{tabular}


Table S4. E. coli bacterial strains and plasmids utilized.

\begin{tabular}{|c|c|c|}
\hline Strains and Plasmids & Features & Source \\
\hline \multicolumn{3}{|l|}{ Strains } \\
\hline BW25113 & rrnB3 $\Delta l a c Z 4787$ hsdR514 $\Delta(\operatorname{araBAD}) 567 \Delta(r h a B A D) 568$ rph-1 & 41 \\
\hline BW25113 $\Delta$ cas2 & $\Delta$ cas $2, \mathrm{Km}^{\mathrm{R}}$ & 41 \\
\hline \multicolumn{3}{|l|}{ Plasmids } \\
\hline pCA24N & $\mathrm{Cm}^{\mathrm{R}} ; \mathrm{lacI}^{q}$ & 42 \\
\hline pCA24N_cas2 & $\mathrm{Cm}^{\mathrm{R}} ;$ lacI $^{q}, \mathrm{P}_{\mathrm{T} 5-\mathrm{lac}}::$ cas2 $^{+}$ & 42 \\
\hline pKD4 & FRT::Kan ${ }^{\mathrm{R}}:: F R T, \mathrm{Amp}^{\mathrm{R}}$ & 38 \\
\hline
\end{tabular}


Table S5. Primers used in this study for qPCR and qRT-PCR. * indicates excision primers.

\begin{tabular}{|c|c|}
\hline Gene & Sequence (5’-3) \\
\hline \multicolumn{2}{|c|}{ Spacer knockout } \\
\hline \multirow[t]{4}{*}{ DS } & F:TTATGCGGATAATGCTACCTCTGGTGAAGGAGTTGGCGAAGGCGTCTTGA \\
\hline & GTGTAGGCTGGAGCTGCTTC \\
\hline & R:CCCCGGTAGATTTGGATGGTTTAAGGTTGGTGTCTTTTTTACCTGTTTGA \\
\hline & CATATGAATATCCTCCTTAG \\
\hline \multirow[t]{2}{*}{ Spacer } & F: GAAGGAGTTGGCGAAGGCGTCTTGA \\
\hline & R: GTTGGTGTCTTTTTTACCTGTTTGA \\
\hline \multicolumn{2}{|l|}{ qRT-PCR } \\
\hline \multirow[t]{2}{*}{ purM } & F: CGGTGTTGATATTGACGCGGG \\
\hline & R: CAGCACGGGTTCACGATATTTTTG \\
\hline \multirow[t]{2}{*}{$y d f D$ (Qin) } & F: TCAGCATTTGTGCTTGTTCTG \\
\hline & R: CTGCCGGGATTTCGATATTA \\
\hline \multirow[t]{2}{*}{ hokD (Qin) } & F: GCCCTGATCGTCATCTGTTT \\
\hline & R: AGCTGTGAAGACAGCGACCT \\
\hline \multirow[t]{2}{*}{ yрjF (CP4-57) } & F: TATGGCCTGACACTGAACGA \\
\hline & R: GTTGTTGCCACGAAAATCCT \\
\hline \multirow[t]{2}{*}{ rzoD (DLP-12) } & F: TGCTCTGCGTGATGATGTTG \\
\hline & R: TCTCTCTGAGGGTGAAATAATCC \\
\hline \multirow{2}{*}{ essD (DLP-12) } & F: CTCACAGTGGGCAGCAATAG \\
\hline & R: TATTCACCTCTCGCAGCCTT \\
\hline \multicolumn{2}{|r|}{ ( } \\
\hline \multirow[t]{2}{*}{ *CP4-57 } & F: AAGCATGTAGTACCGAGGATGTAGG \\
\hline & R: TATGTCTCCTCACCGTCTGGTCGG \\
\hline \multirow[t]{2}{*}{ *e14 } & F: GTGCAAACATCGGTGACGAA \\
\hline & R: TTCAGCAGCTTAGCGCCTTC \\
\hline \multirow[t]{2}{*}{ *DLP-12 } & F: CAAAAGCCATTGACTCAGCAAGG \\
\hline & R: CGGATAAGACGGGCATAAATGA \\
\hline \multirow[t]{2}{*}{ purM 400} & F: CTGATTGCACTCGGTTCCAG \\
\hline & R: CGTTTTCACCGTTGGCATTG \\
\hline \multirow[t]{2}{*}{ Spacer 3-1 } & F: CATGCAATTACAACATCAGGGTA \\
\hline & R: GCCATTGGTAAAACCTTCCA \\
\hline \multirow[t]{2}{*}{ Spacer 3-2 } & F: AAATTCCGAAAAAGCTCCTGA \\
\hline & R: TGCTTTCAAGATTATGGCGTA \\
\hline \multirow[t]{2}{*}{ Spacer 6} & F: CACGAAAGCCAGCCTATTCC \\
\hline & R: CCGCTGTTTCTTTCTCCAGG \\
\hline \multirow[t]{2}{*}{ Spacer 8} & F: GCCAGCATAATGAGATCGGC \\
\hline & R: TTTTACCCAAACTCAGCGCG \\
\hline \multirow[t]{2}{*}{ Spacer 11} & F: TGTGCAGTTGTACCAGTGGA \\
\hline & R: CAACCCAGCAAAGTTTCGGA \\
\hline \multirow[t]{2}{*}{ Spacer 12-1 } & F: TGTATGTGCCCCGGTGTTAT \\
\hline & R: GCAGATGAAGGCGCATTACA \\
\hline \multirow[t]{2}{*}{ Spacer 12-2 } & F: AGCTTTACACCTCGGCTCAT \\
\hline & R: CCGGAACTCTTGTGTTGGTG \\
\hline \multirow[t]{2}{*}{ Spacer 12-3 } & F: TCTACAGGGAAAGGACGACC \\
\hline & R: CTCTGCAACCAAAGTGAACCA \\
\hline \multirow[t]{2}{*}{ Spacer 13} & F: ACAACCACTATCGCCCСTTT \\
\hline & R:GGTAAGGCTGCATTGGGAAG \\
\hline
\end{tabular}




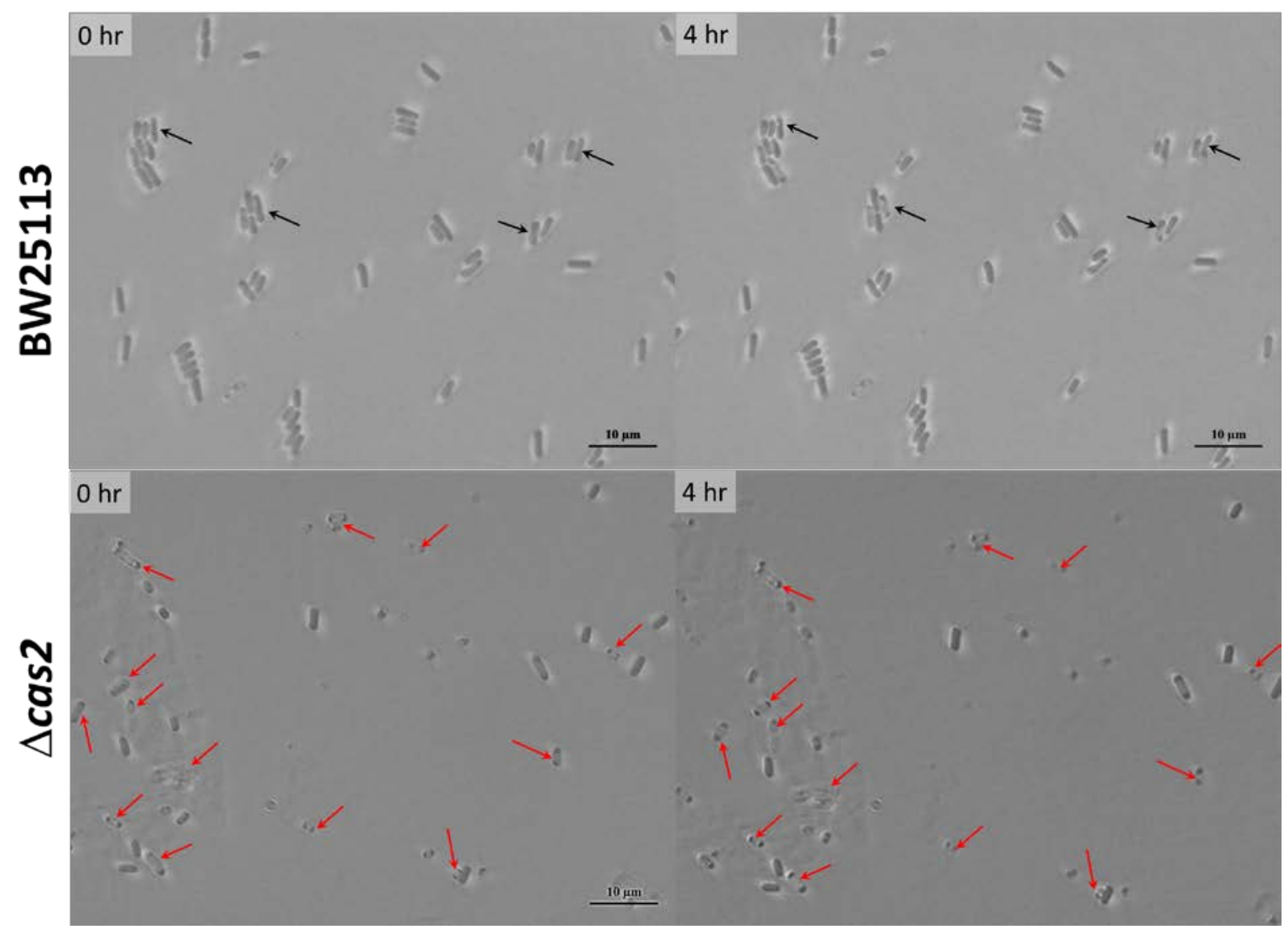

Supplementary Figure 1. Single cell persister waking of BW25113 $\Delta$ cas2. Persister cell waking of BW25113 and BW25113 $\Delta$ cas2 on M9 $0.4 \%$ glucose agar plates incubated at $37^{\circ} \mathrm{C}$ for 4 hours. Black arrows indicate waking cells, and red arrows indicate ghost cells. Scale bars indicate $10 \mu \mathrm{m}$. Representative results from two independent cultures are shown. 
Spacer 3: 5'-GGCTCCCTGTCGGTTGTAATTGATAATGTTGA-' 3

3' -ATGTATGTAGACAGCCAACATAAAGGGAGGTCTTA-' 5 : DLP-12

Spacer 3: 5'-GGCTCCCTGTCGGTTGTAATTGATAATGTTGA-' 3 3'-TTCAGAAGAGACAGCCAAATTACACAGCGGTCTGT-' 5: DLP-12

Spacer 6: 5'-TCAGCTTTATAAATCCGGAGATACGGAAACTA- ' 3 3' -TGTAACGGCAGTCTAACGGCCTCTATGCCCTAGTC-' 5 : CP4-57

Spacer 8: 5' 5 $^{\prime}$-CTGCTGGAGCTGGCTGCAAGGCAAGCCGCCCA - 3 3'-GACGCCGTGGTCGACCGACGTTTCGTGGCGTACAA-' 5: CPS-53

Spacer 11: 5' -CCCGGTAGCCAGGTTTGCAACGCCTGAACCGA-' 3 3' -AGTAAGTCATCGGTCCAAAACTTGTGTAGTTCGCG-' 5 : CP4-6

Spacer 12: 5' -GCAACGACGGTGAGATTTCACGCCTGACGCTG-' 3 3' -GAGTTAGGTAGGCTGTCTAAAGTGCGGCTCGTAAG-' 5: CP4-57

Spacer 12: 5' -GCAACGACGGTGAGATTTCACGCCTGACGCTG-' 3 3' -GGTCGCTGCGTATAGTCCCTAGTGCGGACTGCCTA-' 5 : Rac

Spacer 12: 5' -GCAACGACGGTGAGATTTCACGCCTGACGCTG-' 3 3'-GGTCGCTGCGTATAGTCCCTAGTGCGGACTGCCTA-' 5 : Qin

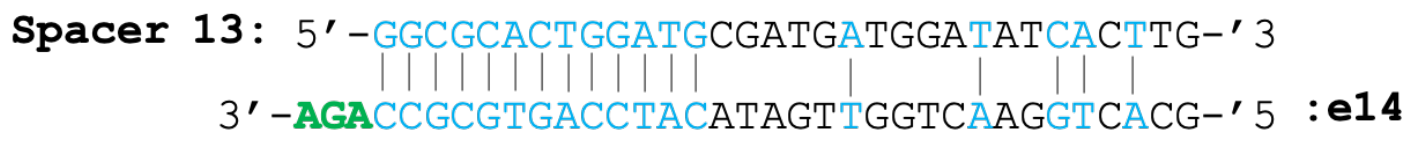

Supplementary Figure 2. Predicted base-pairing interactions between each of the CRISPR spacers (i.e., spacers 3, 6, 8, 11, 12, and 13) and a protospacer within the cryptic phages (DLP-12, CP4-57, CPS-53, CP4-6, CP4-57, Rac, Qin, and e14). Note, some spacers match more than one region within a particular cryptic prophage. The PAMs are indicated by bold/green highlighted text, and yellow highlight indicates active PAMs ${ }^{26}$ (3'TTC; i.e., 5'GAA, is the consensus PAM for $E$ coli). The complementary nt between the spacers and cryptic prophage protospacers is indicated by blue text and vertical lines. 

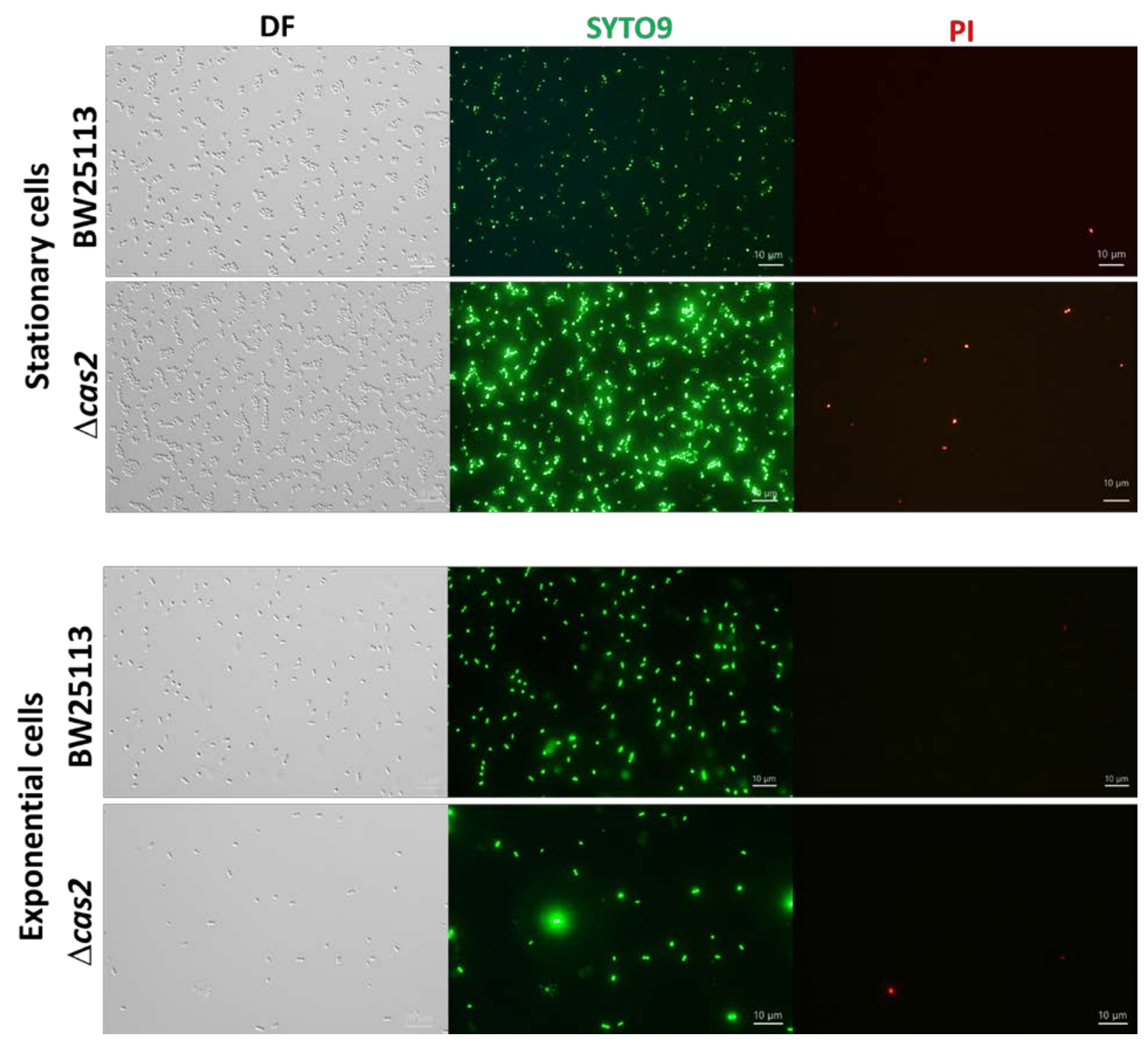

Supplementary Figure 3. LIVE/DEAD staining of stationary (turbidity 2.0) and exponential (turbidity 0.8) cells shows the cas2 mutation causes cell death. DF is dark field, SYTO9 is a membrane permeable stain for nucleic acids (green), and PI is propidium iodide, which is a membrane impermeable stain for the nucleic acids of dead cells (red). Tabulated cell numbers are shown in Table S2. 